Applied Mathematical Sciences, Vol. 11, 2017, no. 20, 975 - 983

HIKARI Ltd, www.m-hikari.com

https://doi.org/10.12988/ams.2017.7275

\title{
On a Manufacturing Capacity Problem in High-Tech Industry
}

\author{
Luca Grosset and Bruno Viscolani
}

Università degli Studi di Padova

Dipartimento di Matematica Tullio Levi-Civita

Via Trieste 63, 35121 Padova, Italy

Copyright (C) 2017 Luca Grosset and Bruno Viscolani. This is an open access article distributed under the Creative Commons Attribution License, which permits unrestricted use, distribution, and reproduction in any medium, provided the original work is properly cited.

\begin{abstract}
We modify the market-clearing price proposed by Pangburn and Sundaresan for an optimal production model in high-tech industry. Under our assumptions the market-clearing price depends also on the demand rate; this hypothesis makes the problem more difficult but returns an optimal production rate which is decreasing in a left neighborhood of the final time, as one would expect for economic reasons. As the solution to the fundamental optimal control problem is characterized through some equations which do not have explicit solutions, we have to solve the capacity problem only numerically. To this purpose we present a numerical procedure to perform a satisfactory, though approximate, analysis of the model.
\end{abstract}

Mathematics Subject Classification: 91B38, 90B30, 49N90, 90-04

Keywords: Production, Capacity, Optimal control, Numerical solution

\section{Introduction}

In the relevant paper "Capacity decisions for high-tech products with obsolescence" [5], Pangburn and Sundaresan describe the capacity decision for hightech products and introduce a special market-clearing price. The idea behind 
this model is that, using the market-clearing price, the production rate and the sales rate are equal. In our personal opinion, two are the drawbacks of this model: first, an exogenous constraint is introduced to define the time horizon; second, the optimal production rate is always constant and equal to the capacity in the whole programming interval. In [4] Grosset and Viscolani try to improve the model analyzing it as a free final time optimal control problem. Moreover, they introduce a quadratic production cost function that makes the optimal sales rate decreasing in a left neighborhood of the final time. Even if quadratic production cost functions are widely used in the economic literature (see e.g. [1]), its introduction in Pangburn and Sundaresan's model may appear lacking of justification, or made on purpose to observe a decreasing optimal sales rate.

Here we focus on a different aspect of Pangburn and Sundaresan's model, namely its demand structure, and try to improve the definition of marketclearing price. In fact, its analytical form, as proposed in [5], is well chosen to represent the obsolescence and market-saturation effects. Nevertheless, it lacks a fundamental feature of an inverse demand (rate) function, namely the dependence on the demand rate. In this paper, we modify the Pangburn and Sundaresan's price function so that the market-clearing price depends on the demand rate. As a consequence we will observe that the optimal production rate becomes decreasing in a left neighborhood of the final time, even with a linear production cost function.

In the final part of the paper we consider the original problem in the context of the new market-clearing price and of the quadratic production costs: we want to find the optimal plant capacity assuming that the capacity costs can be represented by a strictly increasing and convex function, as in [5]. Of course, as the results presented in the first part of the paper are known up to the numerical solution of relevant equations, the solution of the capacity problem is obtained only through numerical computations.

The paper is organized as follows: in Section 2 we introduce a new definition for the market-clearing price and state a production problem as a free final time optimal control problem, following the same approach as presented in [4]. In Section 3 we prove the existence of an optimal solution and characterize it exploiting some results obtained in [4]. In Section 4 we present a numerical approach to solve the capacity problem.

\section{A new definition for market-clearing price}

The main references for our work are the paper by Pangburn and Sundaresan [5], who develop their research in the field of capacity setting, and the paper by Grosset and Viscolani [4], who discuss the dynamic aspects of the problem.

Two recent surveys on a similar issue are the papers by Van Mieghem [8] and 
by Erkoc and $\mathrm{Wu}[3]$.

In this paper we assume that $s(t)$ describes the sales flow at time $t \geq 0$; hence, the function $S(t)$ such that $\dot{S}(t)=s(t)$ and $S(0)=0$ represents the cumulative sales at time $t$. We introduce a new definition of the market-clearing price:

$$
p(t, s, S)=\left\{\begin{array}{lc}
p_{0}(1-t / \alpha)(1-S / \mu)-\beta s & t<\alpha, S<\mu \\
0 & \text { elsewhere }
\end{array}\right.
$$

with $p_{0}, \alpha, \mu, \beta>0$. In this definition:

- $p_{0}$ is the maximum price;

- the factor $(1-t / \alpha)$ represents the obsolescence (time-related) effect;

- the factor $(1-S / \mu)$ represents the market-saturation (volume-related) effect;

- the term - $\beta s$ represents the output rate (current-sales-related) effect, as it is the typical variable term in a linear inverse demand curve (see [2, p.138, 251, 268]).

In the following we assume that a monopolistic firm wants to choose the sales flow $s(t)$ in order to maximize its profit in the programming interval $[0, T]$, where the time horizon $T$ is a decision variable. We recall that (1) represents a market-clearing price, so that $s(t)$ is both sales flow and production rate. As done in the original model [5], we assume that the plant capacity $\rho>0$ is exogenously given and constant in the whole programming interval $[0, T]$. Moreover, we assume that the production cost is quadratic $c(s)=\nu s+\kappa s^{2} / 2$, with $\nu>0, \nu<p_{0}, \kappa>0$. Under these hypotheses the firm wants to solve the following free final time optimal control problem:

$$
\begin{array}{ll}
\max _{s(\cdot) \in L^{2}([0, T],[0, \rho])} & \int_{0}^{T} p(t, s(t), S(t)) s(t)-c(s(t)) d t \\
T \in(0,+\infty) & \\
& \dot{S}(t)=s(t) \\
& S(0)=0
\end{array}
$$

Now it is important to relate this model to other models already analyzed in literature, which lie at the boundary of the model parameter region, with $\beta=0$ (i.e. where the current-sales-related effect is neglected), and with $\kappa=0$ (i.e. where the production cost function is linear). 
1. $\beta=0, \kappa=0$ and $T$ satisfying the constraint $p(T, s(T), S(T))=\nu$ : this is the original Pangburn and Sundaresan's model [5]. The solution is $s^{*}(t)=\rho$ for all $t \in\left[0, T^{*}\right]$ and

$$
T^{*}=\left(\mu+\alpha \rho-\sqrt{(\mu-\alpha \rho)^{2}+4 \alpha \mu \rho \nu p_{0}}\right) / 2 \rho
$$

2. $\beta=0, \kappa=0$ and $T$ chosen to solve problem (2); the problem has the same optimal solution as the previous one (see [4]). This proves that the final time constraint of case 1 is redundant.

3. $\beta=0, \kappa>0$ (quadratic production cost) and $T$ is chosen to solve problem (2); the optimal sales flow and final time are characterized in [4]: it is important to notice that in this instance of the model the optimal sales flow is decreasing in a left neighborhood of the optimal final time as it is seen in real life situations.

Hence, the model with $\beta>0$, which is well justified from a theoretical point of view, has not been studied yet. We close this section observing that the definition (1) may appear unrealistic, because the price is negative at high values of sales flow. Now, although a negative price is certainly not justified from an economic point of view, we will observe that such a scenario does not occur in any optimal solutions. This approach to simplify the analysis of a model is rather common: for example in supply chain models the demand is frequently described as a linear function strictly decreasing in price, therefore for high values of price the demand becomes negative (see e.g. [7]). However, since at the optimality the demand function is always positive, the trouble seems to be rather just a mathematical issue than a modeling problem.

\section{Analysis of the model}

First of all, we want to prove an existence result for a solution to (2).

Theorem 3.1 The free final time optimal control problem (2) admits an optimal solution.

Proof. We apply Filippov-Cesari's existence theorem regarding free final time $[6$, p.145] to problem (2). We observe that all feasible controls take their values in the compact set $[0, \rho]$, and the free final time is chosen in the compact interval $[0, \alpha]$. Moreover, the set of all admissible trajectories is non-empty and uniformly bounded: i.e. for all feasible controls $s(t)$, the associated state function $S(t)$ satisfies $\|S(t)\| \leq \rho \alpha$ for all $t \in[0, \alpha]$. Finally, the function $(t, s, S) \mapsto p(t, s, S) s-c(s)$ (which characterizes the objective functional) 
and the function $(t, s, S) \mapsto s$ (which characterizes the motion equation) are both linear in the state $S$.

In order to find some candidate optimal solutions to problem (2), we use the Pontryagin's necessary conditions. We notice that the objective functional is

$$
\int_{0}^{T}\left[p_{0}\left(1-\frac{t}{\alpha}\right)\left(1-\frac{S(t)}{\mu}\right)-\nu\right] s(t)-\frac{2 \beta+\kappa}{2} s^{2}(t) d t
$$

and we can notice that problem (2) shows the same formal features as the problem studied in [4]. The term $\kappa s^{2}(t) / 2$ accounts for the production cost and the term $\beta s^{2}(t)$ accounts for the current-sales-related effect on price and hence on revenue. These two terms sum up and affect the optimization process in the same way.

In the following, Theorem 3.2 concerns the situation of an over-dimensioned production plant, and provides the definition of a parameter $\zeta$ and of the critical production capacity $\tilde{\rho}$, which are essential to find the solution where the plant capacity is not over-dimensioned. Theorem 3.3 provides the solution in the latter situation. All the results are given without proofs because they follow directly from the analogous results already shown in [4].

Theorem 3.2 Let there exist an optimal solution $\left(s^{*}(t), S^{*}(t), T^{*}\right)$ to the free final time optimal control problem (2) which does not exploit the full capacity $\rho$ at any positive time (i.e. such that $s^{*}(t)<\rho$ for all $t \in\left(0, T^{*}\right]$ ), then the optimal control, state, and final time are unique and have the following analytical form

$$
s^{*}(t)=s_{1}(t ; \tilde{\rho}), \quad S^{*}(t)=S_{1}(t ; \tilde{\rho}), \quad T^{*}=T_{1}(\tilde{\rho})
$$

where, after setting $\zeta=\sqrt{p_{0} / \alpha \mu(2 \beta+\kappa)}$, the functions $s_{1}, S_{1}$ and $T_{1}$ are

$$
\begin{aligned}
s_{1}(t ; \sigma) & =\sigma \cosh (\zeta t)-\zeta \mu \sinh (\zeta t) \\
S_{1}(t ; \sigma) & =\frac{\sigma}{\zeta} \sinh (\zeta t)+\mu(1-\cosh (\zeta t)) \\
T_{1}(\sigma) & =\frac{1}{\zeta} \tanh ^{-1}\left(\frac{\sigma}{\mu \zeta}\right)
\end{aligned}
$$

and $\tilde{\rho} \in(0, \rho]$ is the unique solution to the equation in $\sigma$

$$
p\left(T_{1}(\sigma), 0, S_{1}\left(T_{1}(\sigma) ; \sigma\right)\right)=\nu
$$

Therefore, if $\rho \geq \tilde{\rho}$ (with $\tilde{\rho}$ defined as in the theorem above), then there exists a unique solution to the profit problem (2) which does not exploit the full capacity $\rho$ and this optimal solution is provided in the previous theorem. On the other hand, if $\rho \leq \tilde{\rho}$, then the optimal solution is described in the following theorem. 
Theorem 3.3 If $\rho \leq \tilde{\rho}$ then the unique solution to the free final time optimal control problem (2) has the following analytical form

$$
s^{*}(t)=s_{2}(t ; \tilde{\tau}), \quad S^{*}(t)=S_{2}(t ; \tilde{\tau}), \quad T^{*}=T_{2}(\tilde{\tau})
$$

where

$$
\begin{aligned}
& s_{2}(t ; \tau)= \begin{cases}\rho & t \leq \tau \\
\rho \cosh \zeta(t-\tau)-\zeta(\mu-\rho \tau) \sinh \zeta(t-\tau) & t>\tau\end{cases} \\
& S_{2}(t ; \tau)= \begin{cases}\rho t & t \leq \tau \\
\rho \tau+\frac{\rho}{\zeta} \sinh \zeta(t-\tau)-(\mu-\rho \tau)(1-\cosh \zeta(t-\tau)) & t>\tau\end{cases} \\
& T_{2}(\tau)=\tau+\frac{1}{\zeta} \tanh ^{-1}\left(\frac{\rho}{\zeta(\mu-\rho \tau)}\right)
\end{aligned}
$$

and $\tilde{\tau}$ is the unique solution to the equation in $\tau$

$$
p\left(T_{2}(\tau), 0, S_{2}\left(T_{2}(\tau) ; \tau\right)\right)=\nu
$$

The existence of a unique solution to the necessary optimality conditions stated by Theorems 3.2 and 3.3, together with the existence of an optimal solution stated by Theorem 3.1, guarantees that Theorems 3.2 and 3.3 provide the unique optimal solution of problem (2).

We notice that both conditions (9) and (14), which characterize the critical capacity $\tilde{\rho}$ and time $\tilde{\tau}$, state that the product price equals the marginal production cost at the optimal final time:

$$
c^{\prime}\left(s^{*}\left(T^{*}\right)\right)=\nu=p\left(T^{*}, s^{*}\left(T^{*}\right), S\left(T^{*}\right)\right) .
$$

In case a decision maker wants to choose the capacity level $\rho$, which is assumed here as exogenously given, the problem can be faced only from a numerical point of view, because $\tilde{\rho}$ and $\tilde{\tau}$ can be characterized only as the unique numerical solution of (9), or (14). In the following section we introduce the capacity problem and present a solution algorithm for the optimal capacity level problem.

\section{Capacity problem}

Here we want to show how it is possible to choose the plant capacity using the results obtained in the previous sections. Pangburn and Sundaresan in [5] suggest that the capacity costs can be represented by a strictly increasing and convex function in the form

$$
\rho \mapsto \gamma \rho^{\varepsilon}
$$


where $\gamma>0, \varepsilon>1$. This leads us to study the capacity problem by maximizing the objective function

$$
\rho \mapsto \int_{0}^{T^{*}(\rho)}\left(p\left(t, s^{*}(t ; \rho), S^{*}(t ; \rho)\right)-\nu\right) s^{*}(t ; \rho)-\frac{2 \beta+\kappa}{2} s^{* 2}(t ; \rho) d t-\gamma \rho^{\varepsilon}
$$

We have stressed in the notation that the optimal solution depends on the plant capacity $\rho$. As such dependence is known implicitly through the equations (9) and (14), we propose an approximate approach to solve the capacity problem.

The approximate solution procedure

Step 0 Read the parameters of the free final time optimal control problem, $p_{0}, \alpha, \mu, \nu, \beta, \kappa$, and the ones of the capacity problem, $\gamma, \varepsilon$. Set

$$
\zeta=\sqrt{p_{0} / \alpha \mu(2 \beta+\kappa)}
$$

Step 1 Find $\tilde{\rho}$ by solving the equation $p\left(T_{1}(\sigma), 0, S_{1}\left(T_{1}(\sigma) ; \sigma\right)\right)=\nu$ in $\sigma$. Compute the integral

$$
\tilde{J}=\int_{0}^{T_{1}(\tilde{\rho})}\left(p\left(t, s^{*}(t ; \tilde{\rho}), S^{*}(t ; \tilde{\rho})\right)-\nu\right) s^{*}(t ; \tilde{\rho})-\frac{2 \beta+\kappa}{2} s^{* 2}(t ; \tilde{\rho}) d t
$$

and find the values of the objective function (17) for all $\rho \geq \tilde{\rho}$ :

$$
\rho \mapsto \tilde{J}-\gamma \rho^{\varepsilon}
$$

Step 2 For all $\rho \in(0, \tilde{\rho})$ solve $p\left(T_{2}\left(\tau_{\rho}\right), 0, S_{2}\left(T_{2}\left(\tau_{\rho}\right) ; \tau_{\rho}\right)\right)=\nu$ which is an equation in $\tau_{\rho}$. Compute the integral

$$
J_{\rho}=\int_{0}^{T_{2}\left(\tau_{\rho}\right)}\left(p\left(t, s_{2}\left(t ; \tau_{\rho}\right), S_{2}\left(t ; \tau_{\rho}\right)\right)-\nu\right) s_{2}\left(t ; \tau_{\rho}\right)-\frac{2 \beta+\kappa}{2} s_{2}^{2}\left(t ; \tau_{\rho}\right) d t
$$

and find the values of the objective function (17)

$$
\rho \mapsto J_{\rho}-\gamma \rho^{\varepsilon}
$$

Step 3 Plot the maximum profit function (17) and approximate the function maximum point.

In the final part of the paper we present two plots that are obtained using MATLAB ${ }^{1}$. All the computations are performed by a MacBook Pro equipped with a processor of $2,3 \mathrm{GHz}$ Intel Core i7 and with a RAM of $4 \mathrm{~GB}(2 \times 2 \mathrm{~GB})$, DDR3, 1600MHz. The computation time is 3 seconds. The software used is MATLAB, release R2016b (September 7, 2016: 9.1.0.441655) 64-bit (macl64). The data are set in the following way: $p_{0}=50, \alpha=10, \mu=20, \nu=5, \beta=0.1$, $\kappa=5, \gamma=2, \varepsilon=2$.

\footnotetext{
${ }^{1}$ All the MATLAB files can be freely download at the following ulr: http://www.math.unipd.it/ grosset/resources/MATLAB_files.zip
} 

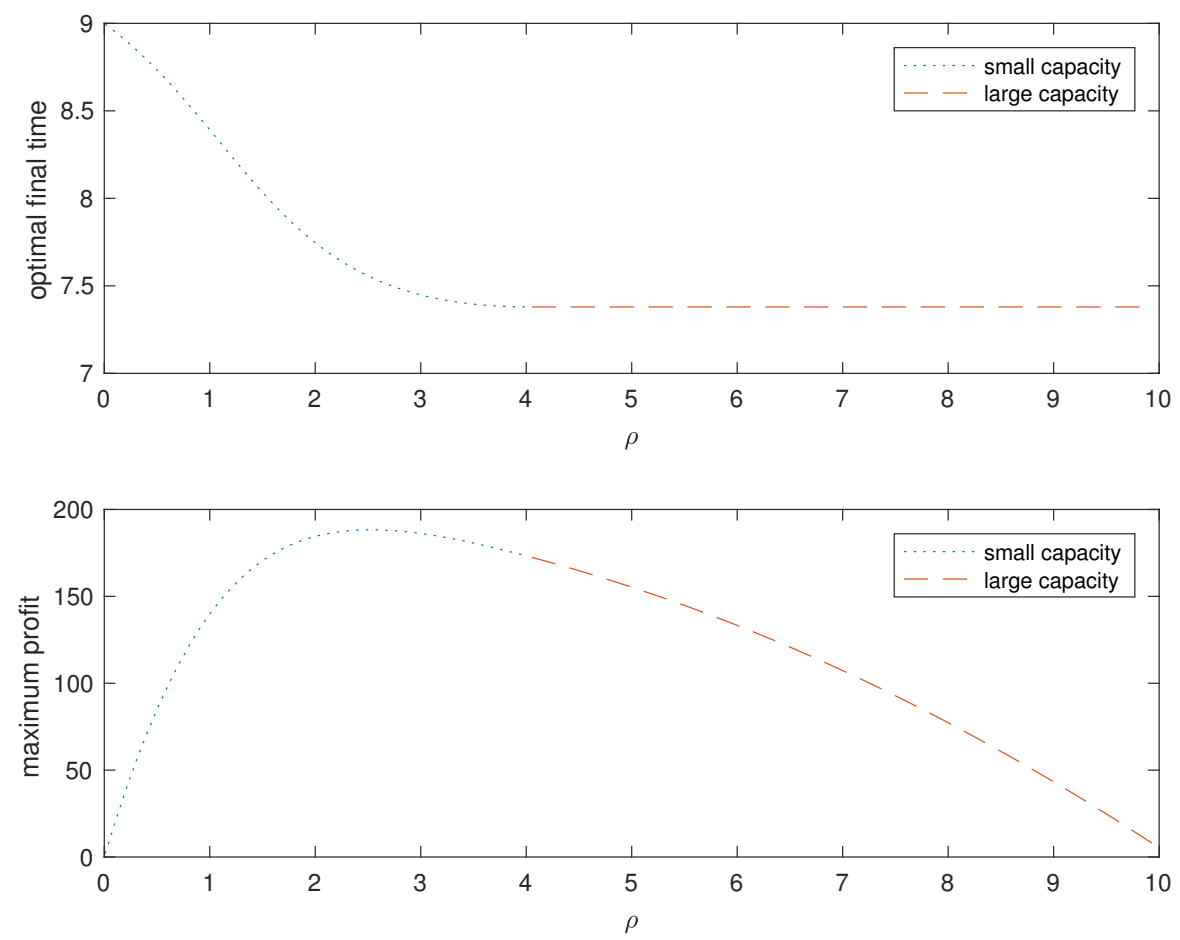

In the first picture we can note that the optimal final time is decreasing in $\rho$ : if the capacity is small, then the programming interval is longer because it takes time to saturate the market. The optimal final time is constant (and it is given by formulas (5) and (9)) when the capacity is greater than the threshold $\tilde{\rho}$ (in this instance $\tilde{\rho}=4.0535$ ).

The second picture represents the profit function (17). The dotted line refers to the small capacity problem (it is found using the results given by Theorem 3.3), while the dashed line refers to the large capacity problem (it is found using the results given by Theorem 3.2). We can notice that the maximum of this function is obtained for a capacity between 2 and 3; the right value is $\rho=2.51$ and can be found simply using MATLAB.

\section{References}

[1] C. Chin-Sheng, Endogenous market boundary and spatial price discrimination with a quadratic production cost function, Letters in Spatial and Resource Sciences, 9 (2016), 157-168.

https://doi.org/10.1007/s12076-015-0149-z 
[2] E. Dockner, S. Jørgensen, N.V. Long and G. Sorger, Differential Games in Economics and Management Science, Cambridge University Press, Cambridge, 2000. https://doi.org/10.1017/cbo9780511805127

[3] M. Erkoc and S.D. Wu, Managing high-tech capacity expansion via reservation contracts, Production and Operations Management, 14 (2005), 232-251. https://doi.org/10.1111/j.1937-5956.2005.tb00021.x

[4] L. Grosset and B. Viscolani, Manufacturing high-tech products subject to rapid obsolescence, Journal of Optimization Theory and Applications, 170 (2016), 993-1008. https://doi.org/10.1007/s10957-016-0928-8

[5] M.S. Pangburn and S. Sundaresan, Capacity decisions for high-tech products with obsolescence, European Journal of Operational Research, 197 (2009), 102-111. https://doi.org/10.1016/j.ejor.2008.05.026

[6] A. Seierstad and K. Sydsæter, Optimal Control Theory with Economic Applications, North-Holland, Amsterdam, 1987.

[7] S.G. Talat and P. De Giovanni, Trade-in and save: A two-period closedloop supply chain game with price and technology dependent returns, International Journal of Production Economics, 183 (2017), 514-527.

https://doi.org/10.1016/j.ijpe.2016.07.012

[8] J.A. Van Mieghem, Capacity management, investment, and hedging: Review and recent developments, Manufacturing and Service Operations Management, 5 (2003), 269-302.

https://doi.org/10.1287/msom.5.4.269.24882

Received: March 9, 2017; Published: April 8, 2017 